\title{
Diseño y validación del cuestionario U-PlanMed para identificación del uso de plantas medicinales en Babahoyo, Ecuador
}

\author{
Design and validation of the U-PlanMed questionnaire for identification \\ of the use of medicinal plants in Babahoyo, Ecuador
}

\author{
Maritza Gallegos-Zurita ${ }^{1, a, b}$, Betty Mazacon ${ }^{1, a, c}$, Luzmila Troncoso $^{2, \mathrm{~d}, \mathrm{e}}$ \\ ${ }^{1}$ Universidad Técnica de Babahoyo - Ecuador. \\ ${ }^{2}$ Universidad Nacional Mayor de San Marcos de Lima - Perú. \\ a Profesora, Facultad de Ciencias de la Salud; ${ }^{\text {B Bóloga; }}{ }^{\mathrm{c}}$ Licenciada en Enfermería; \\ ${ }^{d}$ Profesora Principal, Departamento de Nutrición, Centro de Investigación de Bioquímica y Nutrición, Facultad de Medicina; \\ ${ }^{\text {e } M e ́ d i c o ~ C i r u j a n o, ~ D o c t o r a ~ e n ~ M e d i c i n a . ~}$
}

\begin{abstract}
Resumen
Introducción. El uso medicinal de las plantas, su distribución geográfica, sus formas de preparación, sus formas de administración, entre otros, son cuestiones que todavía buscan respuestas, y no existen en la actualidad instrumentos validados para obtener información. Objetivos. Diseñar y validar un cuestionario para la identificación del uso de las plantas medicinales. Diseño. Estudio descriptivo, observacional, transversal y prospectivo. Lugar. Parroquias rurales de Babahoyo, provincia Los Ríos, Ecuador. Participantes. Jefes de hogar de las comunidades rurales. Métodos. Se diseñó un cuestionario denominado U-PlanMed, el mismo que fue revisado por un equipo multidisciplinario de expertos para determinar su validez lógica y de contenido. También se aplicó a 30 jefes de hogar, en dos momentos, con intervalos de 20 días, durante los meses de enero y febrero de 2015. Se analizaron las respuestas obtenidas de las pruebas de confiabilidad y de validez de constructo, que permitió depurar los ítems, considerando la frecuencia de las opciones de respuesta, su correlación ítem-total y su carga significativa en los ítems durante el análisis factorial. Resultados. El índice de concordancia fue de 97,2\% entre los expertos para la validez de contenido. La confiabilidad del instrumento se midió con el $\alpha$ de Cronbach, siendo su calificación total de 0,82, el índice de fiabilidad 0,98 y el coeficiente de correlación test-retest de 0,96. La medida de adecuación muestral de KMO y prueba de Bartlett resultó 0,8. Conclusiones. El cuestionario U-PlanMed es el primer instrumento utilizado para identificar el uso de las plantas medicinales y las aplicaciones terapéuticas, tiene un alto índice de validez de contenido y de constructo y un nivel aceptable de consistencia, tanto interna como externa.
\end{abstract}

Palabras clave. Estudios de Validación; Cuestionario; Plantas Medicinales; Acción Terapéutica.

\section{Abstract}

Introduction. The use of medicinal plants, their geographical distribution, preparation methods, forms of administration, among others, are issues that require more research and at the moment there are no validated tools or instruments to retrieve this information. Objectives. To design and validate a questionnaire that identifies the use of medicinal plants. Design. Descriptive, observational and transversal study. Location. Rural parishes surrounding the city of Babahoyo, located in the province of Los Rios, Ecuador. Participants. Heads of households in rural communities. Methods. A questionnaire called U-PlanMed was designed and then reviewed by a multidisciplinary team of experts in order to validate its logicality and content. The survey was also administered to 30 different heads of households on two occasions separated by 20-day intervals in the months of January and February 2015. The responses obtained from the validated survey were analyzed which allowed debugging of the items while taking into consideration the frequency of response options, its item-total correlation and significant role on the items during the factorial analysis. Results. The concordance rate was $97.2 \%$ among experts regarding content validity. The reliability of the instrument was measured with Cronbach's Alpha, with a total score of 0.8 , reliability index of 0.98 , coefficient correlation test re-test at 0.96 . The measure of sampling adequacy from KMO and the Barlett test was 0.8 . Conclusions. The U-PlanMed questionnaire is the first tool which can be used to identify the use of medicinal plants and their therapeutic applications. It has a high score of construct and content validity, and an acceptable level of consistency, both internally and externally.

Keywords. Validation Studies; Questionnaires; Plants, Medicinal; Therapeutics.

An Fac med. 2016;77(3):207-12 / http://dx.doi.org/10.15381/anales.v77i3.12399 


\section{INTRODUCCIÓN}

Las poblaciones de América Latina utilizan la medicina tradicional para satisfacer sus necesidades de atención primaria en salud, puesto que, además de ser accesible y asequible económicamente, la medicina tradicional es parte de un sistema más amplio de creencias y tradiciones, constituyéndose en parte integral de la vida diaria y del bienes$\operatorname{tar}^{(1)}$.

En Ecuador, las plantas han sido los principales recursos utilizados en las prácticas de la medicina tradicional por las comunidades campesinas e indígenas. Se estima que $80 \%$ de la población ecuatoriana depende de esta práctica y por consiguiente de las plantas o productos naturales, para la atención de la salud y su bienestar ${ }^{(2)}$.

Actualmente, muchas personas de las zonas rurales todavía dependen de manera directa o indirecta de las plantas para tratar sus enfermedades, pero también para cubrir sus necesidades de alimentación, por cuanto está al alcance de todos y en algunos casos cuentan con sus propios cultivos. En la ciudad su uso es menos frecuente y principalmente está relegado a las personas que habitan en sectores urbano-marginales y de bajos recursos económicos, que les significa menor inversión en el cuidado de la salud. Sin embargo, es muy común encontrar en los mercados de las ciudades ecuatorianas el expendio de gran variedad de plantas medicinales. Esto evidencia que existe la demanda de una población que mantiene la tradición de tratar sus problemas de salud a través del uso de plantas medicinales.

La práctica médica en Ecuador se desarrolla en dos grandes categorías o sistemas: a) una categoría es la medicina ortodoxa, occidental o farmacológica, la cual basa su terapéutica principalmente en sustancias químicas. José Luis Polanco, doctor en Terapia Neural, en 1998, aseguraba que cada vez menos personas utilizan fármacos, siendo uno de los principales motivos los efectos colaterales causados por los químicos ${ }^{(3)}$; b) la otra categoría es la medicina tradicional, conocida también como medicina natural o convencional, que ha sido utilizada por nuestros ancestros y busca la sanación de la persona a través de mecanismos propios del ser humano y de plantas, animales o minerales, sin la utilización de fármacos o sustancias químicas. Esta medicina nació con el ser humano y ha evolucionado, diversificándose en varias ramas o especialidades, entre ellas la fitoterapia, la homeopatía, la terapia neural, la acupuntura, que a su vez tienen subramas y utiliza productos naturales combinados, como refuerzo ${ }^{(3)}$.

Actualmente, la práctica de la medicina tradicional se ha constituido en una política de estado que busca fortalecer y consolidar la salud intercultural, incorporando la medicina ancestral y alternativa al Sistema Nacional de Salud ${ }^{(4)}$. La ejecución de esta política pública está bajo la responsabilidad del Ministerio de Salud, mediante el establecimiento de estrategias para desarrollar, promover y potenciar la práctica de la medicina tradicional, ancestral y alternativa, así como la investigación, para su buena práctica ${ }^{(5)}$.

En este sentido, la autoridad sanitaria nacional es responsable de impulsar el intercambio de conocimientos entre los distintos agentes de la medicina tradicional, fomentando procesos de investigación de sus recursos, diagnósticos y terapéuticos en el marco de los principios establecidos en la Ley, protegiendo los derechos colectivos de los pueblos indígenas y negros o afroecuatorianos, según lo establece el artículo 190 de la Ley Orgánica de Salud ${ }^{(5)}$.

Aun cuando el estado ecuatoriano promueve la investigación en el campo de la medicina tradicional, muy pocos estudios se han publicado en esta área y, específicamente, sobre el uso que se le está dando a las plantas medicinales, a pesar de que es una práctica que se realiza desde nuestros antepasados y que se sigue manteniendo especialmente en las zonas rurales y comunidades campesinas e indígenas.
El uso medicinal que la población rural, en la actualidad, le da a las plantas, su distribución geográfica, sus formas de preparación, sus formas de administración, entre otros, siguen siendo preguntas a responder dentro del estudio básico de la medicina tradicional ${ }^{(6)}$. Esta realidad demuestra que hay muy poca información respecto a protocolos e instrumentos de investigación. De ahí que dentro del programa de Doctorado en Ciencias de la Salud de la Universidad Nacional Mayor de San Marcos de Lima se decidió realizar un estudio que se refiere a las plantas medicinales, sus usos y efectos en el estado de salud de la población rural de Babahoyo, Ecuador, en el año 2015, para el cual se diseñó un cuestionario que permitiera identificar las plantas y las partes utilizadas, el uso que se le da, la finalidad, la forma de preparación, las formas de administración y frecuencia del uso, para el tratamiento de las enfermedades, instrumento que tuvo que ser validado tanto en su contenido como en su construcción.

El cuestionario es la técnica de recolección de datos más empleada en investigación, porque es menos costosa, permite llegar a un mayor número de participantes y facilita el análisis, diseñado para poder cuantificar y universalizar la información ${ }^{(7)}$.

\section{MÉTODOS}

Una vez identificado el propósito de la investigación y definido las variables de estudio dentro de la investigación sobre plantas medicinales -usos y efectos en el estado de salud de la población rural de Babahoyo - Ecuador-, se determinó las dimensiones e indicadores a través de un proceso de operacionalización de las variables, con las cuales se establecieron los criterios a considerar en el diseño y construcción del instrumento de investigación.

El cuestionario se construyó con 6 dominios, expresados en preguntas cerradas y de opción múltiple que estuvieron direccionadas a identificar las 
plantas y las partes utilizadas, las aplicaciones terapéuticas, la finalidad, las formas de preparación, las vías de administración, las dosis y la duración del tratamiento.

Para evaluar la validez lógica y de contenido se consultó a expertos, constituidos por cinco profesionales multidisciplinarios, médicos internistas especializados en naturopatía y homeopatía, antropólogos, sociólogos e investigadores clínicos, quienes evaluaron el instrumento de manera independiente.

Se realizó una prueba piloto con 30 pobladores de la zona rural del Babahoyo, a quienes se les aplicó la primera versión del instrumento para evaluar el nivel de comprensión, legibilidad y reproducibilidad.

Finalizada la revisión del instrumento por el grupo de expertos y efectuada la prueba piloto, se hicieron modificaciones en la redacción de un dominio en donde se ampliaron los ítems y se desagregaron dos dominios, definiendo de mejor manera sus ítems. El instrumento final para su aplicación a la muestra de sujetos contiene ocho dominios con siete ítems referentes a tipos de plantas, tipo de enfermedades, finalidad del uso, partes de la planta utilizada, forma de preparación, formas de administración, dosis y duración del tratamiento.

En la prueba piloto participaron 30 pobladores rurales, adultos de ambos géneros, jefes de hogar encargados del cuidado de la salud de la familia. No se incluyó jefes de hogar de familias dependientes y que compartían la misma vivienda. Fue una muestra no probabilística y su tamaño se calculó considerando la inclusión de 10 sujetos por cada ítem del instrumento, número necesario para efectuar análisis multivariante de factores ${ }^{(8)}$.

Los participantes en el estudio contestaron el cuestionario de manera personalizada con la asistencia del encuestador, puesto que en algunos casos los sujetos solicitaban apoyo para reducir el tiempo de respuesta.
La aplicación del instrumento se realizó acudiendo a cada uno de los hogares de las diferentes sectores rurales del Cantón Babahoyo, en la Provincia de Los Ríos, Ecuador, durante dos semanas, y luego de 20 días se aplicó por segunda ocasión, al mismo grupo de personas; para este momento, los participantes fueron convocados en un aula de la Escuela del Recinto Matecito de la parroquia Febres Cordero.

Para cada momento de administración del cuestionario se utilizó hojas separadas, con las mismas preguntas, estructuradas a partir de los indicadores resultantes de la operacionalización de las variables.

Finalizada la aplicación del instrumento se realizó la depuración de los ítems, especialmente aquellos cuya alternativa de respuesta fue menor al 5\% por la dificultad que se encontró en la comprensión por parte de los encuestados.

Los datos obtenidos de la aplicación del cuestionario fueron analizados estadísticamente con el programa estadístico SPSS vs.19.0. Se determinó la validez de contenido mediante valoración por expertos utilizando la prueba de concordancia entre los jueces (prueba binomial), la validez de constructo con la técnica del análisis factorial, y la confiabilidad del instrumento, es decir, su consistencia interna, mediante el coeficiente alfa de Cronbach. Para determinar la validez externa se utilizó el testretest, coeficiente de Spearman-Brown y dos mitades de Guttman.

\section{RESULTADOS}

Se consideró 35 jefes de hogar, de los cuales $30(85,7 \%)$ respondieron todo el cuestionario, datos que fueron incluidos en el análisis. Cinco jefes de hogar se retiraron y no completaron las respuestas al cuestionario, datos que no fueron considerados.

Participaron 23 mujeres y 7 hombres. La edad de las mujeres comprendió entre 30 y 87 años, media \pm
Tabla 1. Características generales de la muestra, Ecuador $2015(n=30)$.

\begin{tabular}{lcc} 
Características & No. & $\%$ \\
Sexo & & \\
\hline Mujeres & 23 & 76,7 \\
Hombres & 7 & 23,3 \\
\hline Edad (años) & & \\
\hline$>40$ & 2 & 6,7 \\
40 a 59 & 19 & 63,3 \\
60 a 79 & 6 & 20,0 \\
80 o más & 3 & 10,0 \\
\hline Escolaridad & & \\
\hline Hasta $7^{\circ}$ año de educación básica & 19 & 63,3 \\
$10^{\circ}$ año de educación básica & 8 & 26,7 \\
Tercer nivel & 3 & 10,0 \\
\hline
\end{tabular}

desviación $(\mathrm{DE})=54,2 \pm 14,2$ y de los hombres entre 48 y 85 años, media \pm desviación $(\mathrm{DE})=60,6 \pm 11,9$. El 63,3\% de la población apenas tenía nivel de escolaridad básico; eran personas entre 42 y 87 años de edad. Las características demográficas de la muestra se presentan en la tabla 1.

Para contestar el cuestionario, el tiempo que invirtió cada encuestado en la primera aplicación fue en promedio de 20 minutos (rango de 10 a 25 minutos). El intervalo para la segunda administración promedio test-retest fue 20 días (rango 15 a 30 días).

Para la validación de contenido del cuestionario se realizó la prueba de validez por cinco jueces expertos, compuestos por médicos especialistas en naturopatía y homeopatía, antropólogos, médicos clínicos, a quienes se les envió el cuestionario para que emitieran su criterio considerando siete aspectos: pertinencia, concordancia con los objetivos, estructura, concordancia con las variables, secuencia, redacción y número de ítems. La instrucción principal fue calificar cada uno de los ítems del cuestionario considerando los siete aspectos, manifestando los acuerdos con el número 1 (uno) y los desacuerdos con 0 (cero). 
Tabla 2. Valores de $\alpha$ de Cronbach y coeficiente de fiabilidad par-impar del instrumento para medir el uso de las plantas medicinales en las comunidades rurales.

\begin{tabular}{ccccc} 
Ítems & $\begin{array}{c}\text { Media de la } \\
\text { escala si se } \\
\text { elimina el } \\
\text { elemento }\end{array}$ & $\begin{array}{c}\text { Varianza de } \\
\text { la escala si } \\
\text { se elimina el } \\
\text { elemento }\end{array}$ & $\begin{array}{c}\text { Correlación } \\
\text { elemento-total } \\
\text { corregida }\end{array}$ & $\begin{array}{c}\text { Alfa de } \\
\text { Cronbach si } \\
\text { se elimina el } \\
\text { elemento }\end{array}$ \\
Edad/años & 56,50 & 1424,77 & 0,96 & 0,73 \\
Escolaridad & 110,70 & 2546,91 & 0,92 & 0,87 \\
Sexo & 110,40 & 2583,83 & 0,60 & 0,82 \\
Plantas utilizadas & 93,10 & 1228,71 & 0,98 & 0,74 \\
Enfermedad tratada & 93,17 & 1360,14 & 0,98 & 0,73 \\
Finalidad & 110,40 & 2583,83 & 0,60 & 0,82 \\
Partes utilizadas & 108,93 & 2521,65 & 0,77 & 0,81 \\
Preparación & 109,47 & 2345,15 & 0,89 & 0,79 \\
Formas de administración & 110,97 & 2563,34 & 0,60 & 0,81 \\
Dosis/día & 109,77 & 2518,94 & 0,93 & 0,81 \\
Tratamiento & 108,27 & 2491,36 & 0,78 & 0,81 \\
\hline Alfa de Cronbach & & & & 0,82 \\
\hline
\end{tabular}

Una vez tabulados los criterios expresados por los expertos, se realizó la prueba de concordancia (prueba binomial), para establecer el índice de coincidencia entre ellos, observándose que los índices de concordancia fueron de $97,2 \%$, muy significativo para los ocho componentes del cuestionario.

El único desacuerdo encontrado se prestó en el ítem cinco, que se refería a la secuencia, por el juez cinco, sugiriendo que el ítem siete y ocho del cuestionario podría fusionarse, puesto que son complementarios.

Para la confiabilidad, con el fin de determinar el grado en que el instrumento produce resultados constantes y coherentes, se aplicaron las pruebas de consistencia interna y externa ${ }^{(9)}$, obteniendo los siguientes valores:

- Para establecer la consistencia interna del instrumento, se determinó los valores de alfa de Cronbach para el total de ítems del instrumento, observándose una alta consistencia interna de la escala, $\alpha$ de Cronbach $=0,82$, como se describe en la tabla 2 ; de igual manera se determinó el coeficiente de fiabilidad par impar, registrándose un alto índice de fiabilidad por la alta consistencia interna

Tabla 3. Varianza total explicada.
La validación de constructo permite evaluar el grado en que el instrumento refleja la teoría del fenómeno o del concepto que mide, garantizando que las medidas que resultan de las respuestas del cuestionario puedan ser consideradas y utilizadas como medición del fenómeno que queremos medir ${ }^{(10)}$.

Para determinar la validez de constructo se realizó el análisis factorial, en donde se verificó la medida de adecuación muestral KMO (Kayser, Meyer y Olkin) y la prueba de Bartlett. La adecuación de la muestra fue pertinente, presentando un coeficiente de 0,8 y la prueba de Bartlett con $\mathrm{p}=0,00$ y $\mathrm{gl}=$ 28, garantizando que el análisis factorial se realice adecuadamente.

Estos resultados demuestran que tiene sentido aplicar el análisis factorial, para lo cual se optó por la extracción de factores mediante el análisis de los componentes principales y rotación Varimax, arrojando 2 factores primarios que explican el $87,1 \%$ acumulado de la varianza total, como se detalla en la siguiente tabla 3 .

También se pudo detectar que el componente 8 proporcionaba información complementaria al ítem 7 ; este fue eliminado y el ítem siete tomó la denominación de número de administraciones diarias/tiempo de uso. De esta manera, el cuestionario quedó compuesto por 7 componentes, con diferente número de ítems, como se indica en la tabla 4.

\begin{tabular}{|c|c|c|c|c|c|c|c|c|c|}
\hline \multirow[t]{2}{*}{ Componente } & \multicolumn{3}{|c|}{ Autovalores iniciales } & \multicolumn{3}{|c|}{$\begin{array}{l}\text { Sumas de las saturaciones al } \\
\text { cuadrado de la extracción }\end{array}$} & \multicolumn{3}{|c|}{$\begin{array}{c}\text { Suma de las saturaciones al } \\
\text { cuadrado de la rotación }\end{array}$} \\
\hline & Total & $\begin{array}{c}\% \text { de la } \\
\text { varianza }\end{array}$ & $\begin{array}{c}\% \\
\text { acumulado }\end{array}$ & Total & $\begin{array}{c}\% \text { de la } \\
\text { varianza }\end{array}$ & $\begin{array}{c}\% \\
\text { acumulado }\end{array}$ & Total & $\begin{array}{c}\% \text { de la } \\
\text { varianza }\end{array}$ & $\begin{array}{c}\% \\
\text { acumulado }\end{array}$ \\
\hline 1 & 5,69 & 71,10 & 71,10 & 5,69 & 71,10 & 71,10 & 3,73 & 46,59 & 46,59 \\
\hline 2 & 1,28 & 15,95 & 87,05 & 1,28 & 15,95 & 87,05 & 3,24 & 40,47 & 87,05 \\
\hline 3 & 0,57 & 7,11 & 94,17 & & & & & & \\
\hline 4 & 0,19 & 2,38 & 96,55 & & & & & & \\
\hline 5 & 0,17 & 2,09 & 98,64 & & & & & & \\
\hline 6 & 0,07 & 0,86 & 99,49 & & & & & & \\
\hline 7 & 0,03 & 0,37 & 99,87 & & & & & & \\
\hline 8 & 0,01 & 0,13 & 100,00 & & & & & & \\
\hline
\end{tabular}


Tabla 4. Componentes del cuestionario depurado.

\section{Componentes}

¿Qué plantas utiliza usted para tratar y curar las enfermedades?

¿Qué tipo de enfermedad trata con esta planta?

¿Con qué finalidad utiliza?

¿Qué parte de la planta utiliza?

¿De qué forma prepara la parte de la planta utilizada?

¿Cómo aplica la sustancia resultante de la planta al enfermo?

Número de administraciones diarias/ tiempo de uso

\section{$N^{0}$ de ítems}

No definidos

No definidos

2

9

15

8

No definidos

\section{DISCUSIÓN}

El cuestionario U-PlanMed diseñado para identificar el uso de las plantas medicinales en el tratamiento de enfermedades fue sometido a pruebas de validación de contenido, confiabilidad y pruebas de validación de constructo, requisitos esenciales que debe reunir todo instrumento de recolección de datos ${ }^{(11)}$.

La validación de contenido se realizó utilizando la prueba binomial que permitió establecer el índice de coincidencia entre los cinco expertos que participaron en la validación del cuestionario, demostrando que el índice es de $97,2 \%$, siendo muy significativo para los siete de los ocho componentes del cuestionario.

Para determinar la confiabilidad del cuestionario, se validó la consistencia interna obteniendo un alfa de Cronbach de 0,8 , valor muy significativo, encontrándose dentro de los valores recomendados para estos índices que son entre 0,7 y 0,9 (70\% a $90 \%$ ), como lo señalan otros autores ${ }^{(12,13)}$.

Valor de significatividad que fue ratificada mediante el coeficiente de fiabilidad par-impar, registrándose un índice alto de fiabilidad por la alta consistencia interna que se registra entre los ítems pares e impares del instrumento $(\mathrm{rxx}=0,98)$.

La consistencia externa se determinó mediante el coeficiente de correlación test-retest, mostrando un coeficiente de correlación intra-clase de 0,96 y el coeficiente de Spearman-
Brown de 0,98 y dos mitades de Guttman de 0,98 , valores muy significativos que nos permite indicar que el cuestionario es confiable, concordando con otros estudios ${ }^{(14)}$.

La validación de constructo se realizó mediante la aplicación del análisis factorial, pruebas utilizadas en otros estudios para este fin ${ }^{(15-17)}$, verificándose primero la medida de adecuación muestral KMO (Kayser, Meyer y Olkin) con un valor de 0,8 y la prueba de Bartlett obteniendo un valor significativo $(p=0,00)$. Estos resultados garantizan el desarrollo de un análisis factorial adecuado, el mismo que se realizó por la extracción de factores mediante el análisis de los componentes principales y rotación de Varimax, arrojando dos factores primarios que explican el $87,1 \%$ acumulado de la varianza total, lo que queda demostrado que el instrumento es confiable, pues permite que las medidas que resultan de las respuestas del cuestionario pueden ser consideradas y utilizadas para la medición del uso y las aplicaciones terapéuticas de las plantas medicinales.

Los resultados de este estudio demuestran que los criterios definidos en el instrumento diseñado para la identificación de las plantas medicinales utilizadas en el tratamiento de enfermedades en los hogares rurales del cantón Babahoyo en la provincia de Los Ríos, Ecuador, son válidos, puesto que las pruebas de validación cumplen con los criterios de fiabilidad y validez internacionales, lo cual confiere seguridad como un instrumento útil para identi- ficar las especies de plantas medicinales, las formas de uso y las aplicaciones terapéuticas.

Es importante considerar la adaptación semántica del instrumento para su aplicación en los diferentes grupos poblacionales, por el sentido y significado que los temas puedan tener en poblaciones específicas de acuerdo a su visión cultural, influyendo en el análisis de pertinencia de los contenidos de la investigación ${ }^{(18,19)}$.

\section{AGRADECIMIENTOS}

Al Sr. Luis Martínez y Sra. Margoth Agama, líderes comunitarios, por su valiosa colaboración y acompañamiento en el proceso de aplicación del cuestionario, para la recolección de datos.

\section{REFERENCIAS BIBLIOGRÁFICAS}

1. Organización Mundial de la Salud. Estrategia de la OMS sobre medicina tradicional 2002-2005. 2002 [citado 8 de marzo de 2015]; Recuperado a partir de: http://apps.who.int/iris/handle/10665/67314

2. Ansaloni R, Wilches I, León F, Peñaherrera E, Orellana A, Tobar $V$, et al. Estudio preliminar sobre plantas medicinales utilizadas en algunas comunidades de las provincias de Azuay, Cañar y Loja, para afecciones del aparato gastrointestinal. Rev Tecnológica-ESPOL [Internet]. 2010 [citado 23 de mayo de 2015]; 23(1). Recuperado a partir de: http://learningobjects2006.espol.edu.ec/index. php/tecnologica/article/view/40

3. Buitrón X. Ecuador: uso y comercio de plantas medicinales, situación actual y aspectos importantes para su conservación | IUCN Library System [Internet]. 1999 [citado 25 de mayo de 2015]. Recuperado a partir de: https://portals.iucn.org/ library/node/7729

4. Senplades. Objetivo 3. Mejorar la calidad de vida de la población - Plan Nacional 2013 - 2017 [Internet]. 2013 [citado 2 de abril de 2016]. Recuperado a partir de: http://www.buenvivir.gob.ec/objetivo-3.mejorar-la-calidad-de-vida-de-la-poblacion

5. Congreso Nacional, Resolución 423 de 22 de diciembre de 2006. Ley Orgánica de la Salud [Internet]. 2006 [citado 18 de marzo de 2015]. Recuperado a partir de: https://www.google.com/ search?q=La+Ley+Org\%C3\%A1nica+de+la+Sal ud +dice $\% 3 A+$ Art. $+7 \&$ ie $=$ utf-8\&oe $=$ utf-8

6. Lozoya J, Aguilar A, Camacho JR. Encuesta sobre el uso actual de plantas en la medicina tradicional mexicana. Rev Méd IMSS. 1987;25(4):283-91.

7. Arribas M. Diseño y validación de cuestionarios. Matronas Profesión. 2004;5(17):23-9.

8. López-Carmona JM, Rodríguez-Moctezuma R. Adaptación y validación del instrumento de calidad de vida Diabetes 39 en pacientes mexicanos con diabetes mellitus tipo 2. Salud Pública México. 2006;48(3):200-11. 
9. Hernández Sampieri FC. Metodologia de la investigacion de Sampieri - Buscar con Google [Internet]. 2010 [citado 24 de noviembre de 2015]. Recuperado a partir de: https://www.google.com/ search?q=normas + del $+\% \mathrm{C} 3 \% 8 \mathrm{Dndex}+\mathrm{M} \% \mathrm{C} 3 \% \mathrm{~A}$ 9dicus +Internacional $\&$ ie $=$ utf-8\&oe $=$ utf $-8 \# q=$ metod ologia+dela+investigacion+de+sampieri

10. Arribas M. Diseño y validación de cuestionarios. Matronas Profesión. 2004;5(17):23-9.

11. Hernández Sampieri, R., Fernández Collado, C Baptista Lucio, MA. Metodologia de la investigación. Quinta edición. México D.F.: McGRAW-HILL / Interamericana Editores, S.A. DE C.V.; 2010:656 p.

12. Guerrero G, López J, Villaseñor N, Gutiérrez C, Sánchez $Y$. Diseño y validación de un cuestionario de hábitos de vida de alimentación y actividad física para escolares de 8 a 12 años. Revista Chile Salud Pública. 2014;18(3):249-56.

13. Sánchez R, Echeverry J. Validación de escalas de medición en salud. Revista Salud Pública. 2004;6(3):302-18.

14. Márquez-Sandoval YF, Salazar-Ruiz EN, MacedoOjeda G, Altamirano-Martínez MB, Bernal-Orozco
MF, Salas-Salvadó J y col. Diseño y validación de un cuestionario para evaluar el comportamiento alimentario en estudiantes mexicanos del área de la salud. Nutr Hosp. 2014;30(1):153-64.

15. Campos RS. Validación de un cuestionario sobre prácticas de los padres que influyen en la salud bucal e integral de sus hijos. Rev Kiru [Internet]. 2015 [citado 28 de marzo de 2016]; 6(1). Recuperado a partir de: http://www.aulavirtualusmp.pe/ ojs/index.php/Rev-Kiru0/article/viewFile/338/300

16. Vera-Villarroel $P$, Silva J, Celis-Atenas K, Pavez P. Evaluación del cuestionario SF-12: verificación de la utilidad de la escala salud mental. Rev Médica Chile. 2014;142(10):1275-83.

17. Busquets M, Serra R. Validación del cuestionario International Consultation on Incontinence Questionnaire Short-Form (ICIQ-SF) en una población chilena usuaria del Fondo Nacional de Salud (FONASA). Rev Médica Chile. 2012;140(3):340-6.

18. Mora-Ríos J, Bautista-Aguilar N, Natera G, Pedersen D. Adaptación cultural de instrumentos de medida sobre estigma y enfermedad mental en la Ciudad de México. Salud Ment. 2013;36(1):9-18.
19. Sarmiento González P, Botero Giraldo J, Carvajal Carrascal G. Validez de constructo de la Escala de Medición del Proceso de Afrontamiento y Adaptación de Roy, versión modificada en español. Index Enferm. 2013;22(4):233-6.

Artículo recibido el 26 de noviembre de 2015 y aceptado para publicación el 22 de abril de 2016.

Fuente de financiamiento: Autofinanciado.

Conflicto de intereses: Las autoras declaran no tener ningún conflicto de intereses.

\section{Correspondencia:}

Maritza Gallegos-Zurita

Dirección: Ciudadela Universitaria, Calle W Babahoyo - Ecuador.

Correo electrónico: maritzagallegos.005@hotmail. com 